\title{
The AsF+SDF Meta-Environment Documentation Tools for Free!
}

\author{
M. G. J. van den Brand and E. Visser \\ Programming Research Group, University of Amsterdam \\ Kruislaan 403, NL-1098 SJ Amsterdam, The Netherlands \\ email: \{markvdb,visser\}@fwi.uva.nl \\ www: http://adam.fwi.uva.nl/ \{markvdb, visser $\}$ /
}

Introduction Algebraic specifications can be used for the specification of various aspects of programming languages. The executability of the specifications provides not only a mechanism for rapid prototyping, but also tools that can be connected to a programming environment for the language specified.

Over the last 10 years, the programming environments group at CWI and UvA in Amsterdam has conducted research on the generation of programming environments from formal specifications. The main results of this research are:

- The algebraic specification formalism ASF+SDF designed especially for defining the syntax and semantics of (programming) languages $[1,4]$;

- An interactive Meta-environment for the development and prototyping of programming environments for programming languages [5].

An important consideration in the design of the ASF+SDF formalism and Metaenvironment has been the ease of specification of all relevant properties of programming languages: syntax, static semantics, dynamic semantics, transformations, and so on.

The ASF+SDF Formalism ASF+SDF is a combination of the formalisms AsF [1] and SDF [4]. AsF-Algebraic Specification Formalism-supports many-sorted first-order signatures, conditional equations, and modularization. SDF-Syntax Definition Formalism-supports modular definition of lexical and context-free syntax, possibly disambiguated by priorities. In the combination with AsF, the context-free syntax rules in an SDF definition are interpreted as first-order function declarations.

The ASF+SDF Meta-environment The Meta-environment is an interactive environment for the development of ASF+SDF specifications, which can be immediately executed. This is achieved by lazy and incremental generation of scanners and parsers using the SDF parts of the ASF+SDF specification. The equations can be executed as term rewriting systems, which are incrementally updated after modifications in the specifications.

The ASF+SDF Meta-environment has been used in the development of programming environments for several programming languages. To name a few: $\lambda$-calculus, Eiffel, Action Semantics, Pascal, Lotos. It has been applied during the development of some commercial products: modeling of financial products 
and modeling of security constraints for the Dutch Railway Company. It is also used in teaching computer science courses such as Software Engineering and Compiler Construction.

Generation of Documentation Tools A recent extension to the system is a pretty printer generator. Given the SDF definition of a language a pretty printer for this language can be generated [2]. A generated pretty printer can be manually tuned in order to obtain a satisfactory result. The pretty printer works in two phases, it translates the abstract syntax tree of a program into a box expression, which is then translated into text. This approach enables us to obtain documentation tools with little effort for any language for which a specification exists. A translator from boxes to TEX produces code which can be incorporated in $\mathrm{T}_{\mathrm{EX}}$ or $\mathrm{IAT}_{\mathrm{E}} \mathrm{X}$ documents [3]. All components of this tool are specified in $\mathrm{ASF}+\mathrm{SDF}$.

Demonstration In the first part of the demonstration we give an overview of the possibilities and functionality of the ASF+SDF Meta-environment. In the second part the generation of a documentation tool for a specific language is explained and the generated tool is demonstrated by giving some examples.

More Information Information on $\mathrm{ASF}+\mathrm{SDF}$ and the Meta-environment is available through WWW via http://www.cwi.nl/ gipe/[asf+sdf.html]. The metaenvironment is part of the Centaur system. The procedure to get centaur is fully explained on WWW page http://www.inria.fr/croap/centaur/get.html. Approximately $35 \mathrm{Mb}$ of disk space is needed. Installation is possible on SunOS4, Solaris, or Silicon Graphics IRIX4/5. Main memory size should be at least 16MB.

\section{References}

1. J.A. Bergstra, J. Heering, and P. Klint, editors. Algebraic Specification. ACM Press Frontier Series. The ACM Press in co-operation with Addison-Wesley, 1989.

2. M. G. J. van den Brand. Generation of language independent prettyprinters. Technical Report P9327, programming Research Group, University of Amsterdam, Amsterdam, October 1993.

3. Mark van den Brand and Eelco Visser. From Box to $\mathrm{TEX}_{\mathrm{E}} \mathrm{X}$ An algebraic approach to the generation of documentation tools. Technical Report P9420, Programming Research Group, University of Amsterdam, July 1994. Available as ftp://ftp.fwi.uva.nl/pub/programming-research/reports/1994/P9420.ps.Z.

4. J. Heering, P.R.H. Hendriks, P. Klint, and J. Rekers. The syntax definition formalism SDF - reference manual, version 6 December, 1992. Earlier version in SIGPLAN Notices, 24(11):43-75, 1989. Available as ftp: //ftp.cwi.nl /pub/gipe/reports/ SDFManual.ps.Z.

5. P. Klint. A meta-environment for generating programming environments. $A C M$ Transactions on Software Engineering and Methodology, 2(2):176-201, 1993. 